This item was submitted to Loughborough's Research Repository by the author.

Items in Figshare are protected by copyright, with all rights reserved, unless otherwise indicated.

\title{
Regimes of droplet train impact on a moving surface in an additive manufacturing process
}

PLEASE CITE THE PUBLISHED VERSION

http://dx.doi.org/10.1016/j.jmatprotec.2009.10.018

PUBLISHER

(C) Elsevier

VERSION

AM (Accepted Manuscript)

LICENCE

CC BY-NC-ND 4.0

\section{REPOSITORY RECORD}

Fathi, Saeed, Phill M. Dickens, and Farid Fouchal. 2019. "Regimes of Droplet Train Impact on a Moving Surface in an Additive Manufacturing Process". figshare. https://hdl.handle.net/2134/7828. 
This item was submitted to Loughborough's Institutional Repository (https://dspace.lboro.ac.uk/) by the author and is made available under the following Creative Commons Licence conditions.

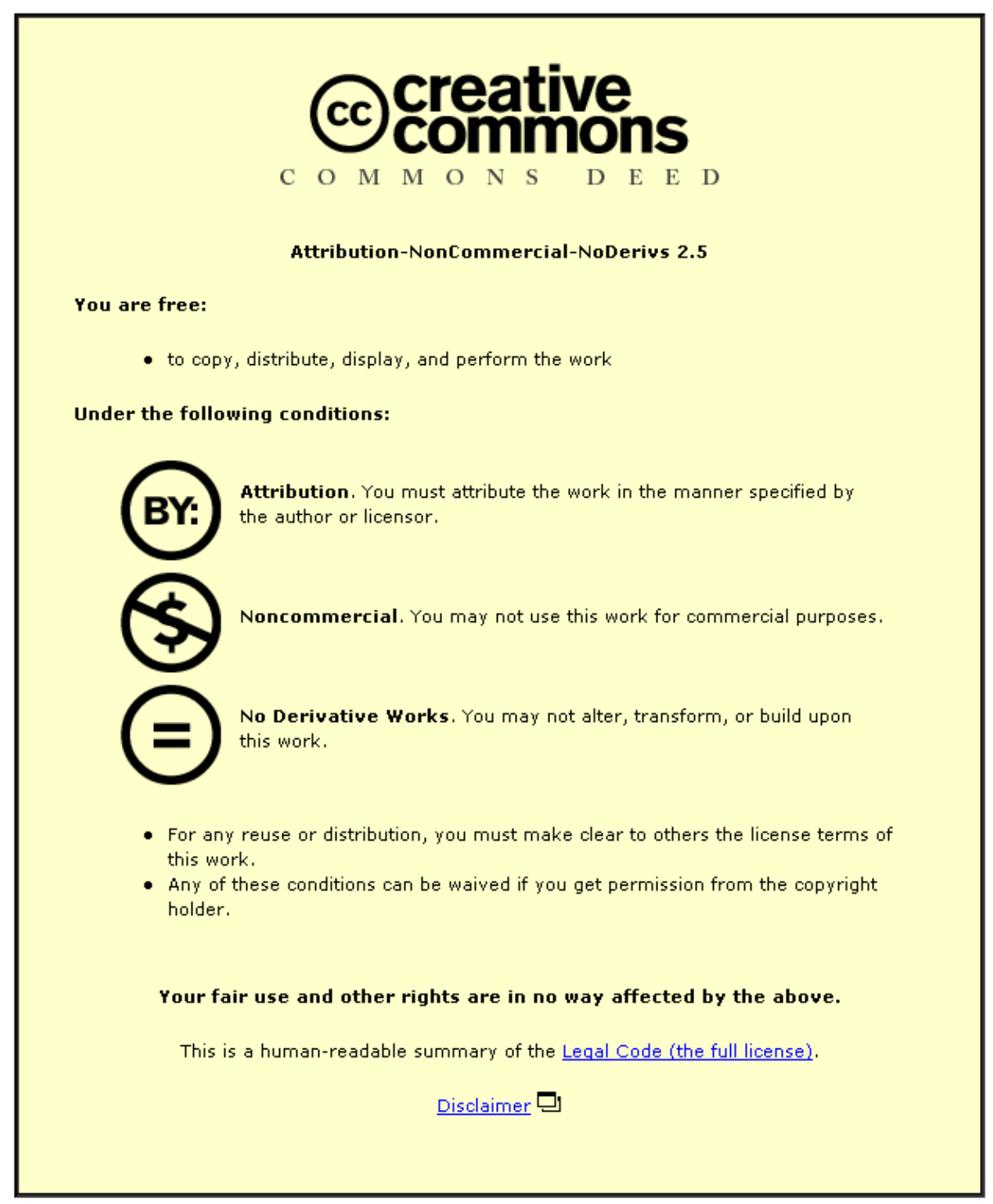

For the full text of this licence, please go to: http://creativecommons.org/licenses/by-nc-nd/2.5/ 


\title{
Regimes of Droplet Train Impact on a Moving Surface in
}

\section{an Additive Manufacturing Process}

\author{
Saeed Fathi*, Phill Dickens, Farid Fouchal \\ Additive Manufacturing Research Group \\ Wolfson School of Mechanical and Manufacturing Engineering \\ Loughborough University \\ Loughborough, LE11 3TU \\ United Kingdom
}

\begin{abstract}
In jetting-based additive manufacturing, one or more droplet trains are deposited on a moving surface to fabricate layers in an additive approach. The impact behaviour of the droplets onto the surface defines the final fabricated layer properties in terms of geometry and surface finish. This paper reports on such behaviour when depositing a solution of a bio-degradable resin toward an ultimate goal of fabricating bone implants in an additive manufacturing process. The solution was jetted via a fixed single nozzle continuous piezoelectric printhead on a moving surface. The effect of two main process parameters, jetting frequency and substrate linear velocity, on the impact behaviour was investigated. Quantitative analysis was undertaken to investigate the droplet formation characteristics and droplet/surface interactions. The phenomena associated with the interaction at the front of the advancing liquid layer was correlated with the process conditions. The result was a classification of the droplet train/moving surface interactions into three main regimes depending on the droplet impingement characteristics and the surface motion.

* Corresponding author. Tel.: +44 1509227 568; Fax: +44 1509227549.

E-mail address: S.Fathi@lboro.ac.uk or Saeed.Fathi@gmail.com (S. Fathi).
\end{abstract}


Keywords: Impact Regimes; Droplet Spreading; Moving Surface; Additive Manufacturing

\section{Introduction}

In droplet-based industrial processes including ink-jet printing, spray coating, etc, wherein liquid droplets impinge onto a surface, it is important to understand the interaction between the droplets and the surface. However, the interaction accompanies a number of issues such as mechanical interaction as well as heat transfer or phase change. These provide challenging on-going aspects of research in understanding the whole phenomena which assist the process optimisation.

Jetting technology has been applied to develop a number of processes in an additive layer approach to manufacture parts rapidly. Hopkinson et al. introduced some of the processes which have been commercialised and Calvert (2001) reviewed the materials delivered by the ink-jet technology for manufacturing applications. A layer of material is deposited via jetting on top of a previously spread solidified layer in an additive manner to build a three-dimensional shape. In such an application, a train of droplets impinge onto a moving substrate to make beads of materials as a part of a whole layer when depositing many beads side by side.

In many droplet-based processes, continuous generation of a droplet train is based on pressurising fluid to make a stream and then vibration of a piezoelement inside a fluid channel through which the stream is made as reported by Gritts et al. (2006). The piezoelement is stimulated by a continuous voltage signal wave in a preset waveform, frequency and amplitude. By vibrating the element, a contraction and expansion of the volume inside the channel forms a pressure wave propagating towards a nozzle at the end of the channel. The pressure waves propagated into the stream through the nozzle generates a capillary wave which finally disintegrates the 
stream into tiny uniform droplets depending on the amplitude of the wave. In continuous mode jetting a train of droplets is made consequently to either impinge onto a surface or be recirculated by a deflection system for an on-demand deposition on the surface as reported in a review paper by Le (1998).

Frohn and Ross (2000) and Rein (2002) reviewed the extensive literature published on the impact behaviour of droplets on different surfaces. More recently, Yarin (2006) showed in his review that according to a droplet's physical properties and the surface conditions, it may stick to the surface, bounce off or splash and split into smaller droplets as a general classification of the impact behaviour in case of a stationary surface. In the case of a stationary surface, a droplet will impact and spread radially to form a liquid layer which is usually called a lamella. Further phenomena after spreading depends on the physical and kinematical properties of the droplet mainly expressed by the dimensionless numbers of Weber $(W e)$, Reynolds $(R e)$ and Ohnesorge (Oh). Yarin and Weiss (1995) showed numerically and experimentally that a high Weber number can result in splashing of a droplet in which a crown may be formed whereas with a low Weber number, a uniform deposition is achieved. The study was on the effect of droplet velocity on impact behaviour with a liquid film as a wet surface formed by previous spreading droplets. They showed that at a low impact velocity, capillary waves were generated on the thin liquid film and were similar to each other. In addition, formation of a crown at higher impact velocities and secondary droplets from the splashing behaviour were investigated in their study in which the crown formation was analytically shown to be a result of a kinematic discontinuity. Later, Rioboo et al. (2003) undertook extensive experimental research to detail the crown formation behaviour in the case of a single droplet impact onto a stationary wet surface. They studied the threshold impact conditions between crown 
formation, splashing and spreading behaviour and showed a combination of the dimensionless film thickness and the dimensionless numbers of Weber and Ohnesorge are needed to be taken into account for the threshold condition.

Despite the many papers published for the case of impact on a stationary surface, few papers have reported the impact behaviour in the case of a moving surface. Mundo et al. (1995) studied droplet impact on a rotating dry disc to find the spreading-splashing threshold in correlation with different Reynolds and Ohnesorge numbers. By varying the rotating surface velocity in their study, the effective impingement angle was calculated to study its effect on the impact behaviour. Although there are a number of papers on the angled impingement (oblique impact) of single aqueous droplet onto a surface, the effect of surface velocity has not been studied independently in detail. Leneweit et al. (2005) studied the oblique impact of a single droplet onto a deep fluid with an impact angle ranging from about $5^{\circ}$ to $65^{\circ}$ and reported the mechanisms for capillary wave, lamella formation and the partial immersion of impinging droplet into the target fluid depending on the impact condition. Okawa et al. (2008) also investigated the impact of single water droplet onto a stationary water surface at different impingement angle and focused on the formation behaviour of the secondary droplet in the splashing condition. A similar study to Mundo et al. (1995) by Chen and Wang (2005) on water droplets impinging onto a rotating cylindrical disk, reported the effect of different tangential velocities on impact behaviour. The tangential velocity was achieved by combining the rotational speed of the disk and an angled impingement based on which a tangential Weber number was introduced. Qualitative research of Courbin et al. (2006) also showed how the impact behaviour is affected by the surface motion where the droplet 
deformation is asymmetric. All of these studies were accomplished with liquids having a viscosity lower than $3 \mathrm{mPa}$.s.

In the jetting-based additive layer manufacturing processes, the impact behaviour at the front of the bead being deposited by the droplet train and the surface motion is of importance for final layer geometry and surface finish. The bead front advances on the surface and it is of interest to study any splash or material build-up in the advancing front which can affect the final quality of the manufactured part.

There is a lack of literature on studying droplet train impact behaviour onto a moving surface. It has been proved in the above citations that an asymmetric droplet deformation is induced by the tangential impact velocity component introduced by either surface motion or angled impingement (or a combination of both) which can be accompanied by splashing at higher impact dynamics. However the studies did not investigate impact of a train of micron size droplets of higher viscosity liquids impinging at high frequencies onto a linear moving surface where a bead is being formed with an advancing front. This paper reports such a situation in which the front of the depositing bead is the focus. Impinging droplets at different frequencies onto an initially dry smooth surface with a range of velocities created different impingement conditions of dry, wet and half dry-half wet surfaces to categorise the impact behaviour quantitatively applying high speed imaging and image analysis.

\section{Experiments}

To study the droplet impact behaviour on a moving surface, the physical properties of the jetting material and the surface condition needed to be known which are covered in this section. Also, the experimental setup applied consisting of a 
continuous jetting system, a moving substrate as a layer deposition system and a machine vision system for droplet impact behaviour monitoring are described.

\subsection{Material and Equipment}

A bio-degradable resin supplied from TNO Science and Industry (the Netherlands) was mixed with isopropanol (Propanol-2 PUR - Acros Organics) to make a diluted solution of $50 \%$ by volume of the solvent (volumetric ratio of $1: 1$ ) as the jetting material for this study. The resin was based on a biodegradable polyester oligomer under development by DSM of the Netherlands for medical application in additive manufacturing processes. Table 1 shows the physical properties of the solution. The pure resin and isopropanol had a density of 0.78 and $1.18 \mathrm{~g} \mathrm{~cm}^{-3}$ respectively. A contact angle measuring instrument (OCR20 - Data Physics Instruments $\mathrm{GmbH}$ ) was used to measure surface tension of the solution with the pendent drop method. The contact angle was measured on a microscope glass slide (Lames Porte-Object - Menzel Glaser GmbH) using the sessile drop method. The dynamic viscosity of the material was measured using a standard rotational viscometer (VT500 - Haake) and the results are shown in figure 1. The result indicates that the solution had a non-Newtonian behaviour at lower shear rates but with very high shear rates in the jetting process (in the order of $10^{4} \mathrm{~s}^{-1}$ ), the viscosity was considered to be approaching a constant value of $25 \mathrm{mPa}$ s for calculating the Weber and Reynolds numbers.

\begin{tabular}{|c|c|c|}
\hline Property & Value & Unit \\
\hline Density & $0.98 \pm 0.01$ & $\mathrm{~g} \mathrm{~cm}^{-3}$ \\
\hline Surface Tension & $26.4 \pm 0.2$ & $\mathrm{mN} \mathrm{m}^{-1}$ \\
\hline Contact Angle & $22.8 \pm 2$ & Degree \\
\hline
\end{tabular}

TABLE 1. Physical properties of the jetting material 


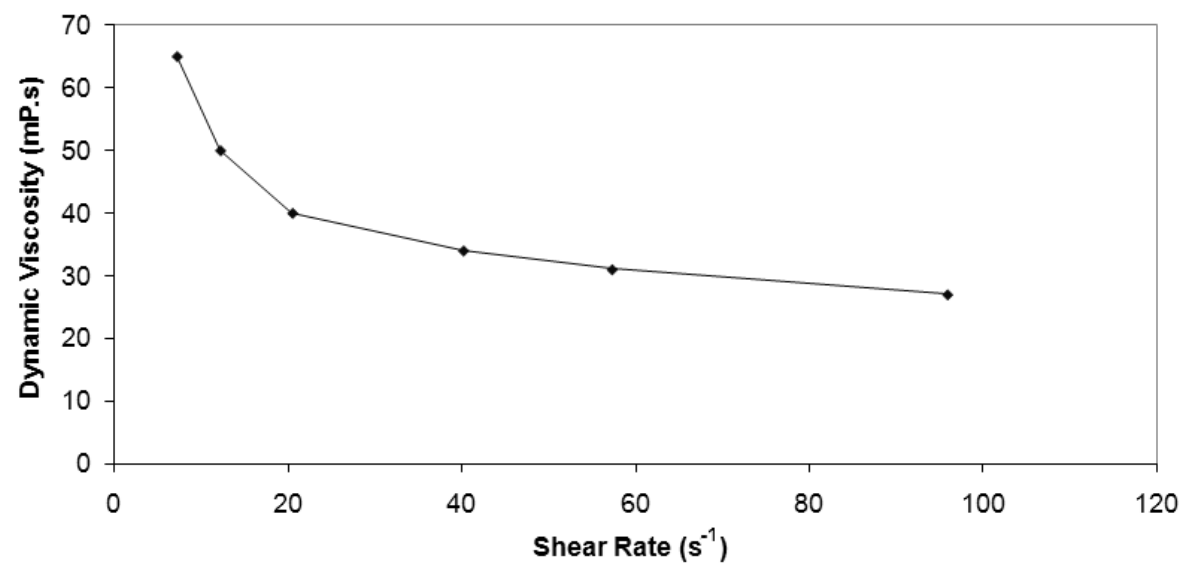

FIGURE 1. Dynamic viscosity of the jetting material at $20^{\circ} \mathrm{C}$

Figure 2 shows a schematic of the applied setup in the current work. This consisted of three main sections namely the jetting system, three-dimensional (3D) motion system and imaging system. The jetting system had an open loop circulation to feed the jetting head from a beaker containing the material. Figure 3(a) shows the circulation system used in the experiments. A prismatic pump (MasterFlex S/L Easyload II) supplied the material through a beaker to a dosing pump (micro-pump) (Knauer HLPC K-120). A damper was used to smooth the flow rate and pressure generated by the pumps. The fluid was consequently pushed by the pumps to the jetting head with an accurate control over the flow rate. The continuous jetting head was piezo-based and manufactured by TNO Science and Technology with an $80 \mu \mathrm{m}$ laser drilled nozzle. The piezoelement of the jetting head was vibrated by an amplifier (LVPZT E-501-00 - Physik Instrumente (PI) GmbH) which received voltage signals from a function generator (TG330 - TTI, Inc.).

The imaging system consisted of a high speed camera (FASTCAM APX-RS Photron Inc.) and a light source (ELSV-60 - Everest VIT). The light source was positioned in order to capture a shadow of the droplets and impact phenomena. A lens was used to magnify the impact location of the droplets on the surface. Positioning of 
the droplets was achieved by a linear stage (LMA-264R-1000 - Aerotech Inc.) in a 3D motion system where the stage provided a variable speed up to $9 \mathrm{~m} \mathrm{~s}^{-1}$. The jetting head was set at a height of $45 \mathrm{~mm}$ above the surface which was a $1 \mathrm{~mm}$ thick glass slide (Lames Porte-Object - Menzel Glaser GmbH) fixed to the linear carriage. Imaging of the droplet impact was achieved on $35 \mathrm{~mm}$ of the length of the glass surface. Figure 3(b) shows the position of the jetting head, camera and the light source relative to the glass surface on which droplets impinged.

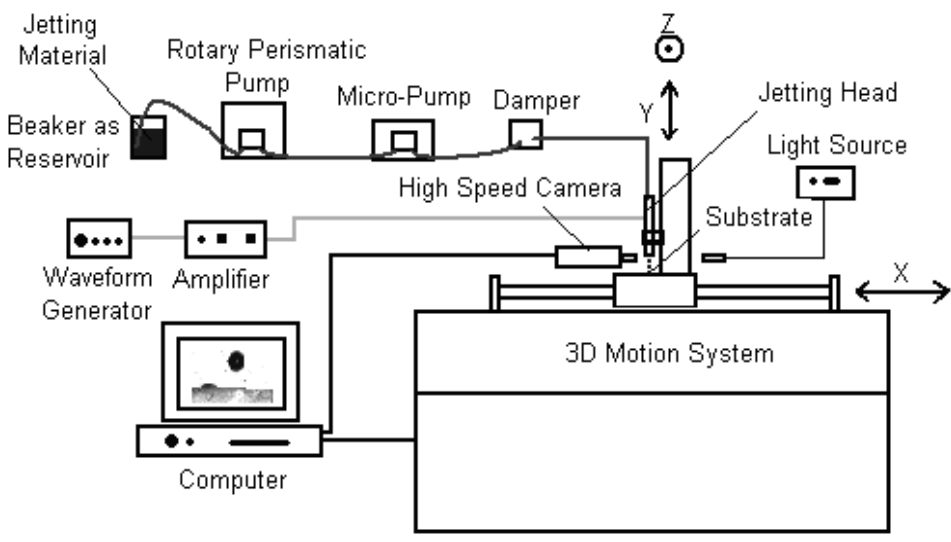

FIGURE 2. A schematic of the setup
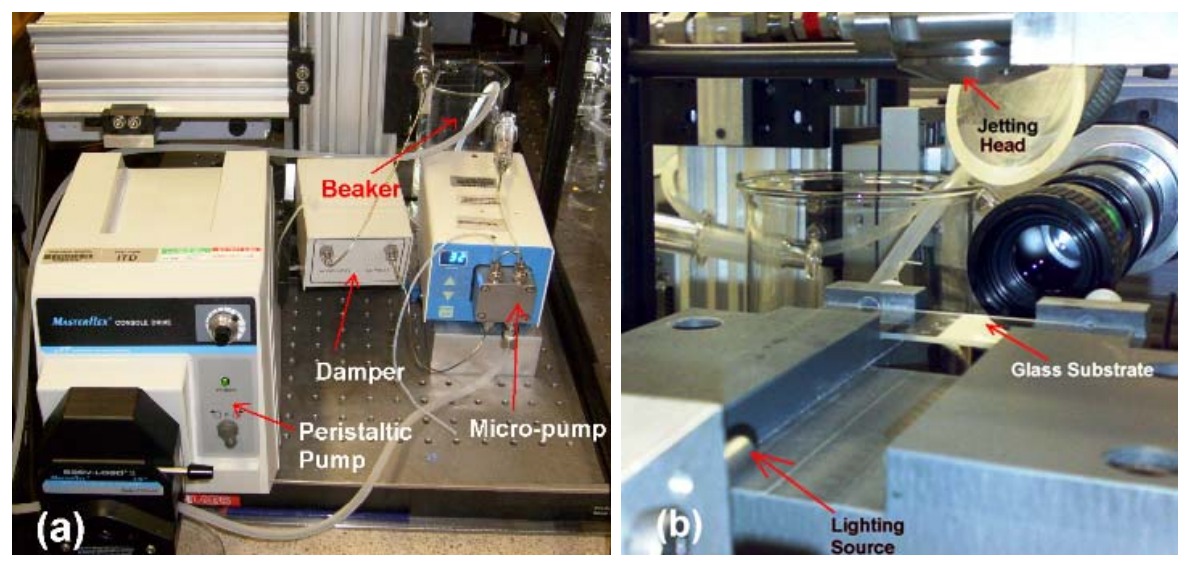

FIGURE 3. ( $a$ ) The circulation system and $(b)$ the imaging system

\subsection{Procedure}


When running the jetting system before piezoelement stimulation, dripping occurred through the nozzle. While dripping, the function generator signals were sent to the amplifier to vibrate the piezoelement in the jetting head. By vibration of the element, the dripping flow of the material was broken down to a train of tiny droplets based on the Rayleigh concept of stream break-up proposed by Rayleigh (1878). Different frequencies (from 5 to $20 \mathrm{kHz}$ ), with a fixed amplitude of $0.65 \mathrm{~V}$, were applied to study the effect of droplet formation on the impact phenomena. The appropriate frequency for the study was found to be between 9 to $18 \mathrm{kHz}$ with a consistent droplet formation. Two frequencies of 12 and $18 \mathrm{kHz}$ were typically selected as the low and high range frequencies in the droplet impact study. All the experiments were undertaken in a controlled room temperature of $20^{\circ} \mathrm{C}$.

The imaging system was set to capture the impact phenomena with an appropriate imaging and shutter speed found to be 45,000 fps (frames per second) and $2 \mu \mathrm{s}$ respectively. The motion programme was run to produce a line of material on the glass substrate. Surface velocities in the range of $1 \mathrm{~m} \mathrm{~s}^{-1}$ to $9 \mathrm{~m} \mathrm{~s}^{-1}$ were applied. Two smaller values of velocity, $0.1 \mathrm{~m} \mathrm{~s}^{-1}$ and $0.5 \mathrm{~m} \mathrm{~s}^{-1}$, were also examined. The camera was triggered to capture the impact of the droplets on the moving surface. The images were then analysed to quantify the material behaviour during impact. Characteristics such as droplet size, velocity and impingement rate along with the impact behaviour such as spread layer thickness (bead height) and phenomena associated with the droplet deformation in the advancing front of the depositing layer were measured using the glass slide’s thickness as a scale.

\section{Results}


Results are presented in two parts of droplet formation characteristics and impact behaviour on the moving surface. The correlation between the two is discussed in the next section.

\subsection{Droplet Formation Characterisation}

Figure 4 shows a sequence of a droplet in flight just before impinging onto the moving surface. The size of droplet and their velocity was measured just before impingement. The droplet velocity was calculated from the displacement of droplet in flight between two consecutive frames as shown in Figure 4.

Figure 5 shows the effect of frequency on droplet size (diameter), velocity and impingement rate. The actual flow rate was also calculated from the droplet formation characteristics to see the consistency of the material supply through the pumping system. At least five jetting trials were accomplished for each jetting frequency with very consistent results in droplet formation characteristics but the mean value was considered for the graphs shown in figure 5 .

By increasing the frequency, the droplet size decreased while in contrast, the droplet impingement rate was increased with frequency. There was a slight variation in droplet velocity despite the fixed voltage signal amplitude applied. This could be a result of the characteristics of the jetting head in expelling droplets. Therefore, from the graphs, the dominant variations in droplet formation were the droplet size and the impingement frequency. 


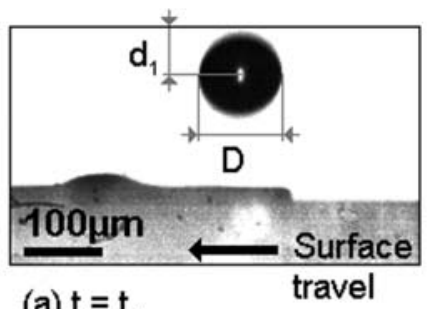

(a) $\mathrm{t}=\mathrm{t}_{0}$

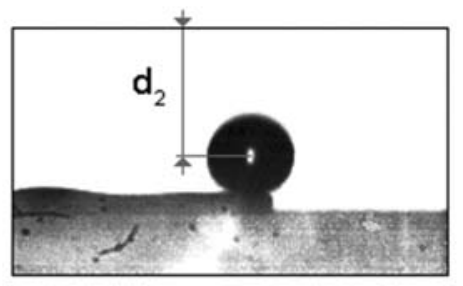

(b) $t=t_{0}+22$ us

FIGURE 4. Measurement of the droplet size and velocity (Jetting frequency: $12 \mathrm{kHz}$ just before impinging onto the surface)

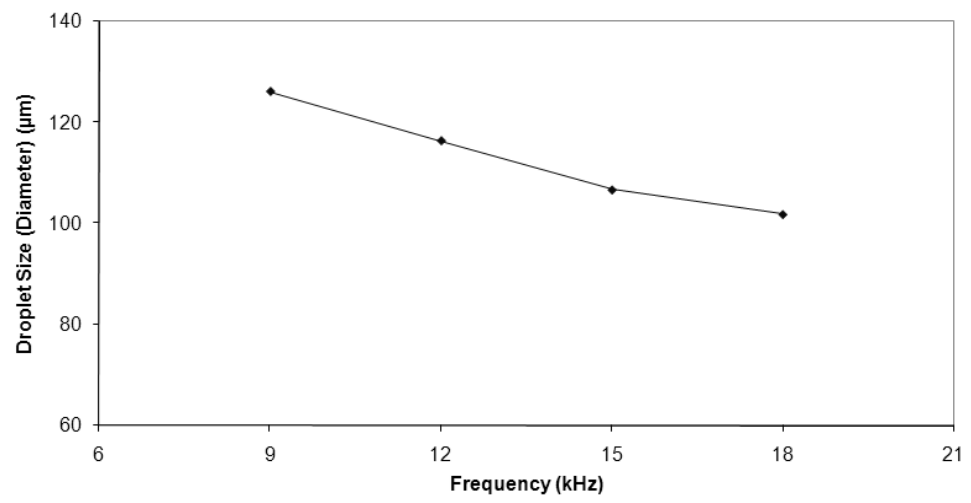

(a)

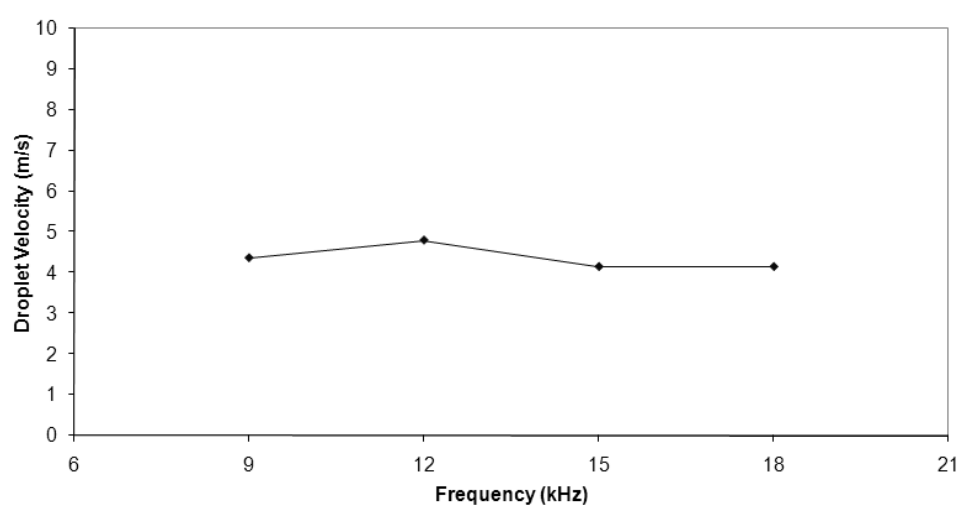

(b)

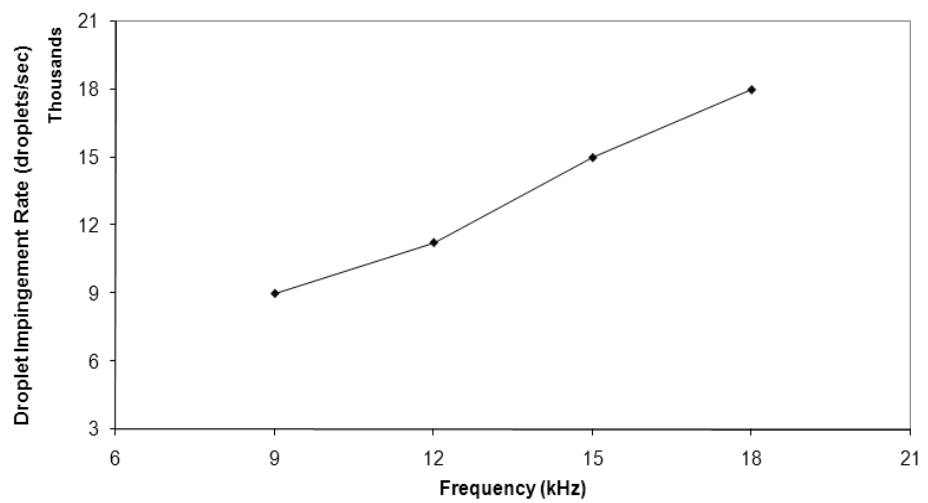

(c) 


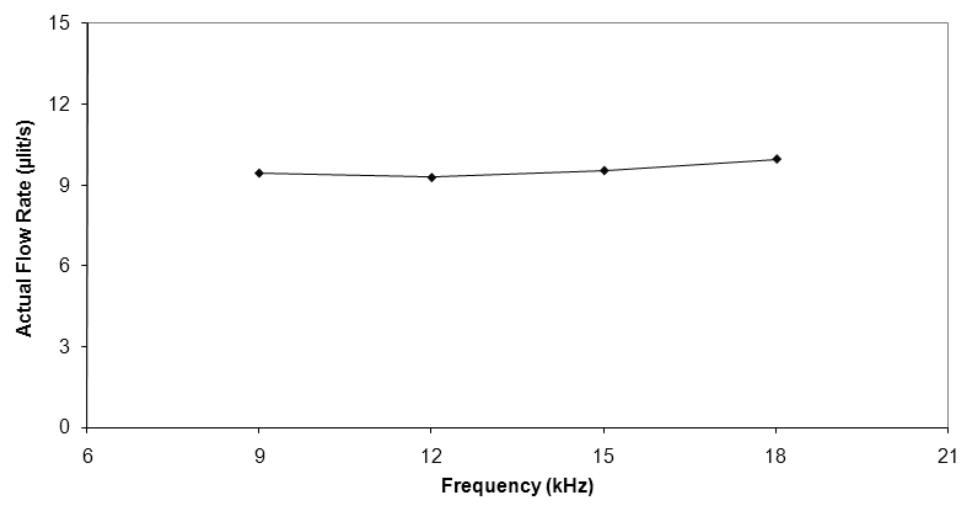

(d)

FIGURE 5. Variation of ( $a$ ) droplet size (diameter) $(b)$ velocity $(c)$ impingement rate, and $(d)$ actual flow rate by jetting frequency

\subsection{Droplet Train Impact on a Moving Surface}

\section{Parameters associated with droplet train and surface motion:}

The interaction of the impinging train of droplets onto the moving surface was found to be affected by the droplet impingement rate, its volume and the surface motion. These factors were considered by taking the material linear deposition rate into account which was the volume deposited per displacement unit of the surface. This gave a unit value of pico-litre per micro-metre $\left(\mathrm{pl} \mu \mathrm{m}^{-1}\right)$. It is obvious that increasing the velocity decreases the linear deposition rate. However, to have a better analysis of impact behaviour, the variation of the linear deposition rate with surface velocity was demonstrated in figure 6. It is seen that the deposition rates at different frequencies (typically shown for 12 and $18 \mathrm{kHz}$ ) are similar as a result of their actual flow rate which was kept constant for all trials. The rate for the very low surface velocity, $0.1 \mathrm{~m} \mathrm{~s}^{-1}$, was too high at both frequencies (93 and $100 \mathrm{pl} \mu \mathrm{m}^{-1}$ for 12 and $18 \mathrm{kHz}$ respectively) to show in the graph of figure 6 . 


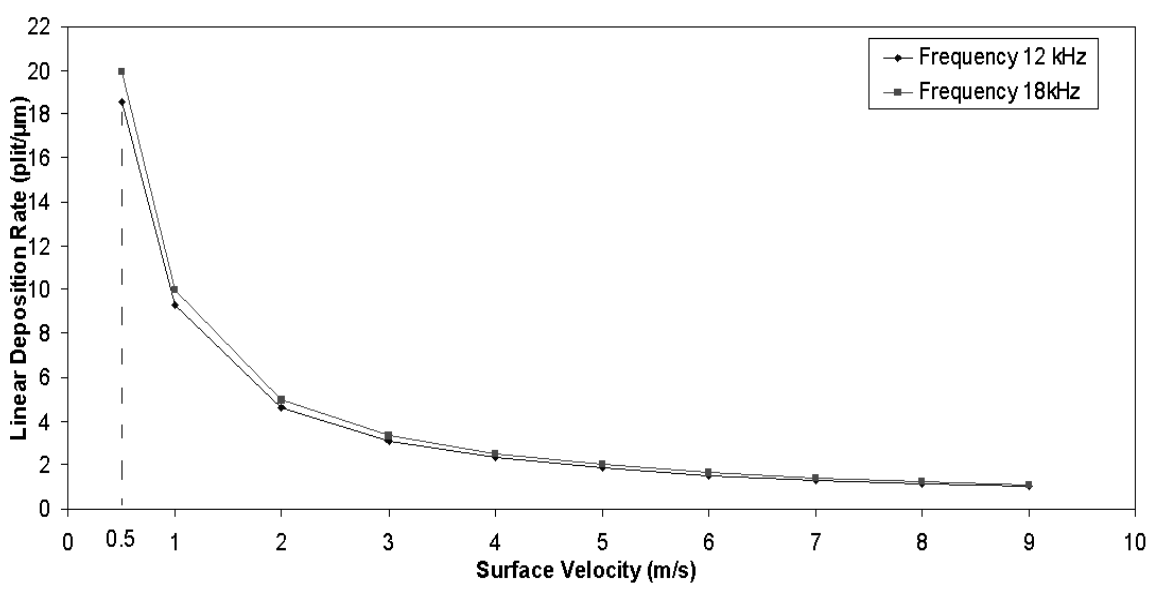

FIGURE 6. Variation of the linear deposition rate with surface velocity in jetting the solution for two typical frequencies

The kinetics of a droplet mainly affects the droplet deformation process in the case of a stationary surface (as discussed in the introduction section). However, a new parameter containing the droplet impingement rate should be taken into account in case of a train of droplets. Therefore, besides the impact energy of a single droplet, the rate of impact energy for the droplet train was also considered. This was determined by multiplying the kinetic energy of one droplet $\left(E=1 / 2 m v^{2}\right)$ by the impingement rate which is equal to droplet generation frequency of the jetting head (figure $5(c)$ ). Also $m$ is the mass of a droplet (figure $5(a)$ ), and $v$ is the droplet velocity (figure $5(b)$ ). The dimensionless numbers of Weber and Reynolds were also calculated for a better understanding of impinging droplets characteristics although only one material was investigated in this study. These numbers are respectively defined as the ratio of inertia forces to surface tension or viscous forces: $W e=\rho D V^{2} / \sigma$ and $\operatorname{Re}=\rho D V / \mu$, where $V$ is the droplet velocity on impact, $D$ is the droplet diameter, $\rho$ as the liquid density, $\sigma$ as droplet surface tension and $\mu$ as the dynamic viscosity which was assumed constant at the very high shear rates of jetting and impingement. A typical droplet deformation at impact on the surface was captured in 3 frames of the 
high speed imaging giving a period of $1 / 15 \mathrm{~ms}$ (shear rate of $1.5 \times 10^{4} \mathrm{~s}^{-1}$ ) which will be discussed later. The Ohnesorge number which defines the interaction between Weber and Reynolds numbers was also calculated based on the equation:

$$
O h=W e^{1 / 2} / \operatorname{Re}\left(\text { or } O h=\mu /(\rho \sigma D)^{1 / 2}\right)
$$

Table 2 shows the trend of impinging droplet train characteristics at different frequencies. Increasing the frequency decreased the impact energy of each individual droplet. The impact energy rate however did not have a similar trend and $12 \mathrm{kHz}$ gave the highest value. The Weber and Reynolds numbers were generally low for the range of frequency studied as a result of the micron sized droplets with relative low velocities and high viscosity compared with previous research reviewed by Yarin (2006). With the relatively high viscosity of the jetting material used in this study, a higher Ohnesorge number was obtained.

\begin{tabular}{|c|c|c|c|c|c|}
\hline $\begin{array}{c}\text { Frequency } \\
(\mathrm{kHz})\end{array}$ & $\begin{array}{c}\text { Impact Energy } \\
(\mathrm{nJ})\end{array}$ & $\begin{array}{c}\text { Impact Energy } \\
\text { Rate }(\mathrm{nJ} / \mathrm{ms})\end{array}$ & $\begin{array}{c}\text { Weber } \\
\text { Number }\end{array}$ & $\begin{array}{c}\text { Reynolds } \\
\text { Number }\end{array}$ & $\begin{array}{c}\text { Ohnesorge } \\
\text { Number }\end{array}$ \\
\hline 9 & 9.7 & 87 & 87.8 & 21.2 & 0.441 \\
\hline 12 & 8.8 & 99 & 93.8 & 21.1 & 0.460 \\
\hline 15 & 5.7 & 85 & 71.1 & 17.6 & 0.479 \\
\hline 18 & 4.4 & 80 & 61.6 & 16.0 & 0.490 \\
\hline
\end{tabular}

TABLE 2. Impact characteristics the droplet train at different frequencies

\section{Qualitative Results:}

Figure 7 shows a sequence (nine consecutive frames) of droplets impinging on the moving dry surface with a relatively low deposition rate and a mid range frequency and high surface velocity. The number of the impinging droplet (e.g. droplet 1 as $D 1$ ), the frame number and the surface travel direction are specified. The time elapsed between two consequent frames was $22 \mu$ s based on the imaging frequency for all the impact sequences shown throughout this paper. As seen, the imaging speed was 
chosen so that the camera captured at least three images for each droplet. The sequence in figure 7 shows impingement behaviour of the three consecutive droplets to illustrate the similarity and repeatability of the impact behaviour at the chosen conditions. As can be seen in figure 7, the spreading droplets made a liquid layer (film) with a specific thickness as a part of a bead being deposited onto the surface. The advancing liquid layer front was a result of the deposition process made by the surface travel. A wave formed on the liquid layer (shown as Wl-Wave 1) caused by impact of droplet 1 and $W 2$ made by droplet 2 are shown in figure 7 . It is seen that the waves shape and motion are similar.

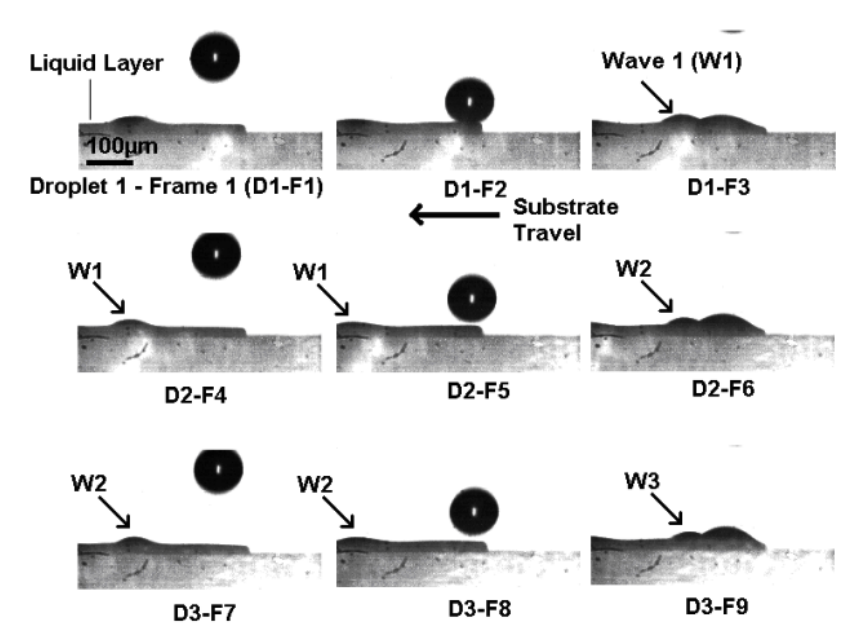

FIGURE 7. The solution's droplets impinging on the surface with a low linear deposition rate (1.2 $\mathrm{pl} \mu \mathrm{m}^{-1}$ ) (Surface Velocity: $8 \mathrm{~m} \mathrm{~s}^{-1}$, Frequency: $15 \mathrm{kHz}$ )

A low linear deposition rate resulted in no wave in front of the advancing liquid layer as seen in figure 7 whereas a high linear deposition rate resulted in formation of a crown like wave in front of the advancing liquid layer as shown in figure 8 . This situation was made at lower jetting frequencies with low surface velocities. The crown-like wave in figure 8 occurred at the interface between the wet/dry surface boundary (shown in the figure) of the advancing liquid layer. The existence of such 
interface as the source of the waves will be discussed later. However, all droplets did not make such a wave as can be seen in frame number 7 to 10 ( $F 7$ to $F 10$ ) of figure 8 showing impact of droplet number 2 (D2). In fact droplet number 2 in frame number 6 (D2-F6), damped the previous droplet generated wave as seen in D2-F7. There was then a periodic behaviour in wave formation in front of the advancing liquid layer.

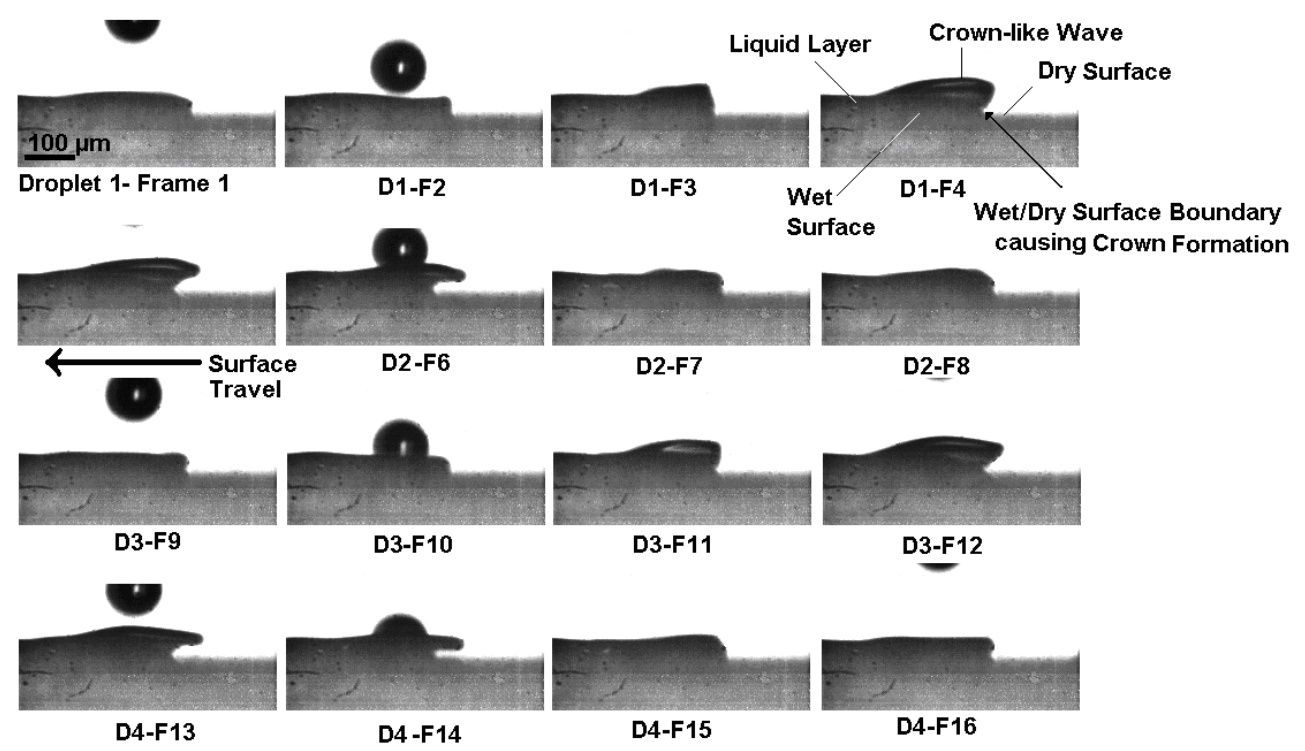

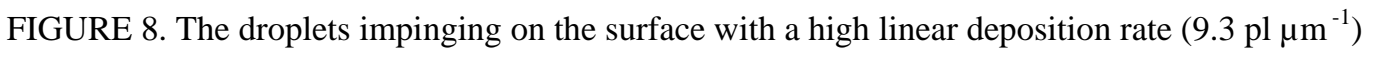
(Surface Velocity: $1 \mathrm{~m} \mathrm{~s}^{-1}$, Frequency: $12 \mathrm{kHz}$ )

Figure 9 shows an un-wetted (un-covered) section of the substrate caused by a very low linear deposition rate. Figure 10, on the other hand, shows the formation, growth and advancement of a wave towards the dry surface and finally its separation from the advancing liquid layer front. This phenomenon seems to be a result of material build-up in the advancing liquid layer front. As shown in the sequence in figure 10, the wave was separated from the advancing front after reaching a certain volume. This phenomenon which will be discussed further was only seen at higher surface velocities and jetting frequencies where the impingement frequency was high. The separation of the built-up liquid from the advancing layer front was repeated 
typically three times along the monitored length of the surface (35 mm). With lower surface velocities typically lower than $3 \mathrm{~m} \mathrm{~s}^{-1}$, the wave diminished before the growth stage.

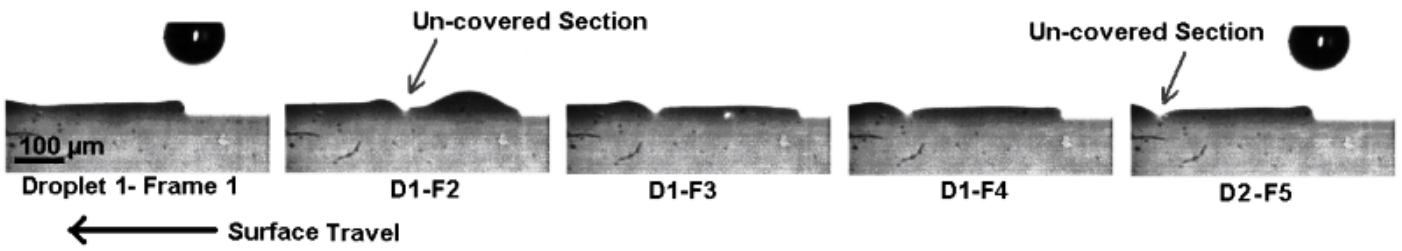

FIGURE 9. Uncovered sections in the droplet spreading at too low linear deposition rate

$\left(1 \mathrm{pl} \mathrm{m}^{-1}\right)$ (Surface velocity: $9 \mathrm{~m} \mathrm{~s}^{-1}$, Frequency: $9 \mathrm{kHz}$ )

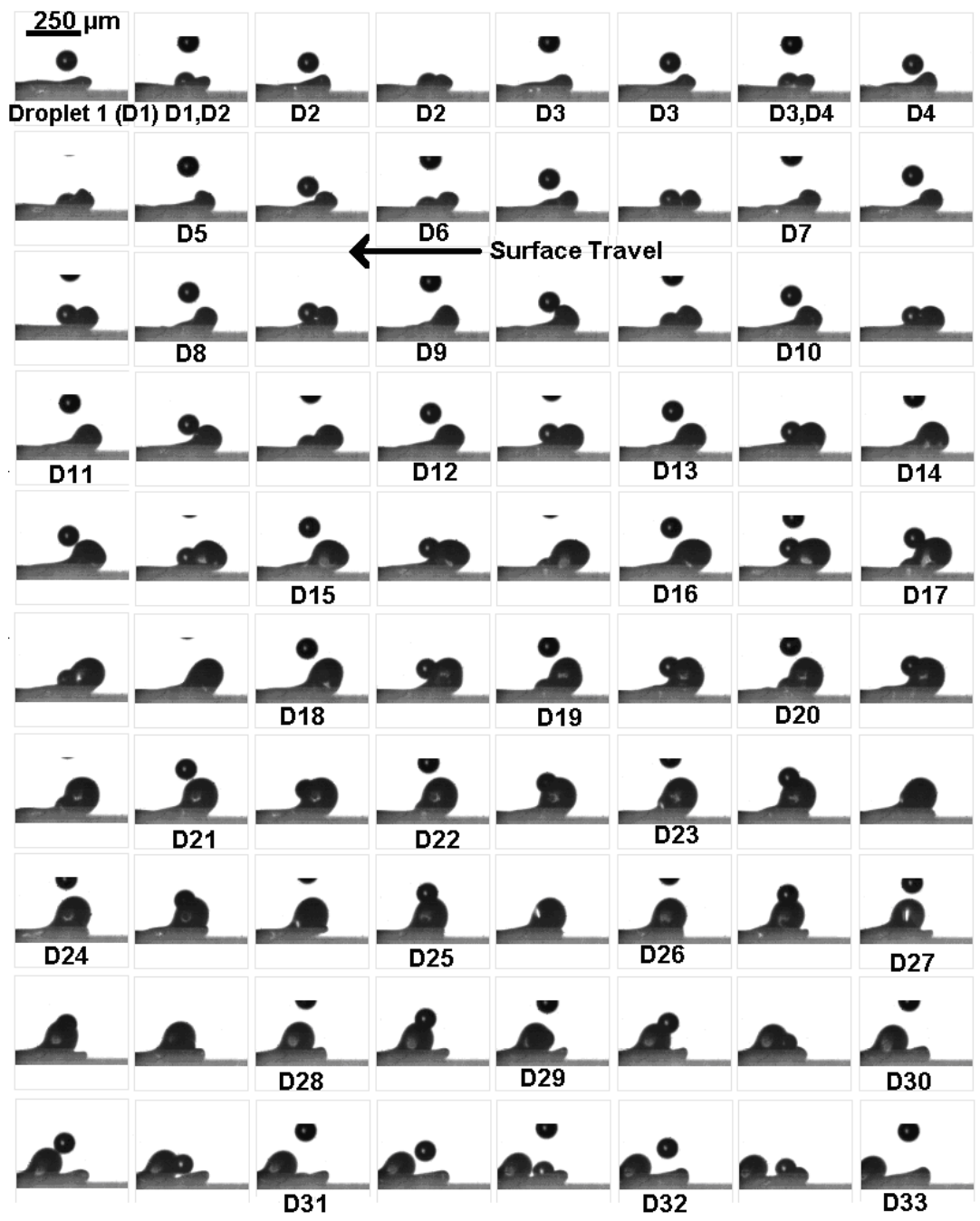

FIGURE 10. Solution liquid build-up wave in front of the advancing liquid layer at a high impingement frequency (Substrate velocity: $7 \mathrm{~m} \mathrm{~s}^{-1}$, Frequency: $18 \mathrm{kHz}$ ) 
From the qualitative analysis of the result, there were two main jetting frequency zones where spreading behaviour was different at higher surface velocities which can be compared typically by looking at figures 7 and 10 . For jetting frequency equal or below $15 \mathrm{kHz}$, there was smooth spreading whereas with a jetting frequency higher than $18 \mathrm{kHz}$, formation of the build-up wave was observed. Therefore, $12 \mathrm{kHz}$ was selected to represent the lower range and $18 \mathrm{kHz}$ the higher range jetting frequencies for the quantitative analysis. Higher jetting frequencies than $18 \mathrm{kHz}$ was of interest for the authors, but due to jet instability at such frequencies, the study could not be extended further (the frequency range for a stable jet was from 9 to $18 \mathrm{kHz}$ as mentioned earlier).

\section{Quantitative Analysis:}

Figures 11 and 12 show variations of the spread layer thickness and the height of the waves formed at the front of the advancing liquid layer by varying surface velocity at two typical jetting frequencies. Dots in the figures indicate the spread layer thickness while the height of the waves is shown by vertical lines on top of the dots to show the maximum height of the waves made above the spread layer thickness. An image is shown inside figures 11 and 12 to indicate how the maximum height of the waves was measured in relation to the layer thickness. By increasing the velocity, the spread layer thickness decreased to a minimum value (at a velocity of $3 \mathrm{~m} \mathrm{~s}^{-1}$ ) for both frequencies after which it remained constant. A thicker layer was generally made when jetting at the higher frequency $(18 \mathrm{kHz})$ despite the smaller droplets. In addition, decreasing the velocity from $3 \mathrm{~m} \mathrm{~s}^{-1}$ to $0.1 \mathrm{~m} \mathrm{~s}^{-1}$ caused an increase of 2 to 3 times in the layer thickness for both frequencies. 
The height of the waves depended on the process conditions as figures 11 and 12 reveal. High values of maximum wave height (seen in figure 12) occurred at the higher frequency $(18 \mathrm{kHz})$ with higher surface velocities $\left(>3 \mathrm{~m} \mathrm{~s}^{-1}\right)$. This corresponds with the situation shown in figure 10 where a growing wave travelling with the advancing liquid layer is seen. However, lower values of maximum wave height seen in figures 8 and 11 occurred at lower droplet frequency and surface velocity where droplets impinged onto the wet substrate and made a crown-like shaped wave at the front of liquid layer. In addition, situations shown in figures 7 and 9, where no wave was occurred with high surface velocities and lower droplet frequency are shown in figure 11.

The built-up waves formed at higher frequencies. The number of droplets to form a wave was observed for surface velocities greater than $3 \mathrm{~m} \mathrm{~s}^{-1}$. The size of the wave formed was considered as the maximum height as shown in figure 12. No specific trend can be seen in table 3 in terms of the number of droplets to form the wave even though the maximum height as shown in figure 12 increased with the surface velocity.

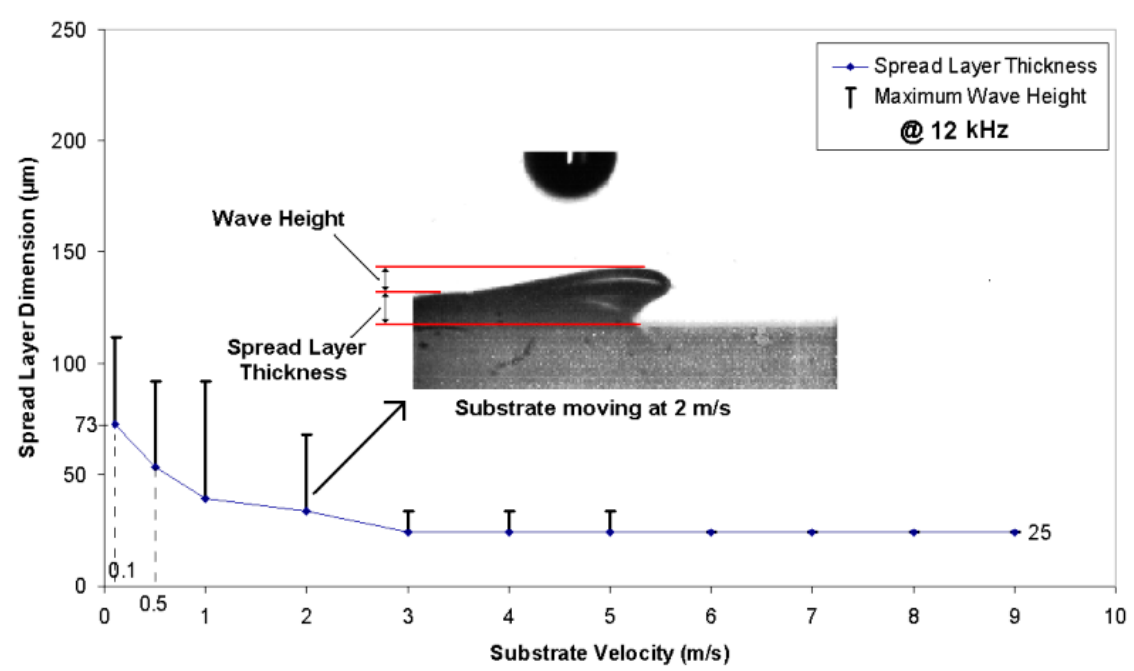

FIGURE 11. Variation of the spread layer dimensions (spread layer thickness and maximum wave height) formed by the droplets jetted at $12 \mathrm{kHz}$ impinging onto the surface with varying velocity 


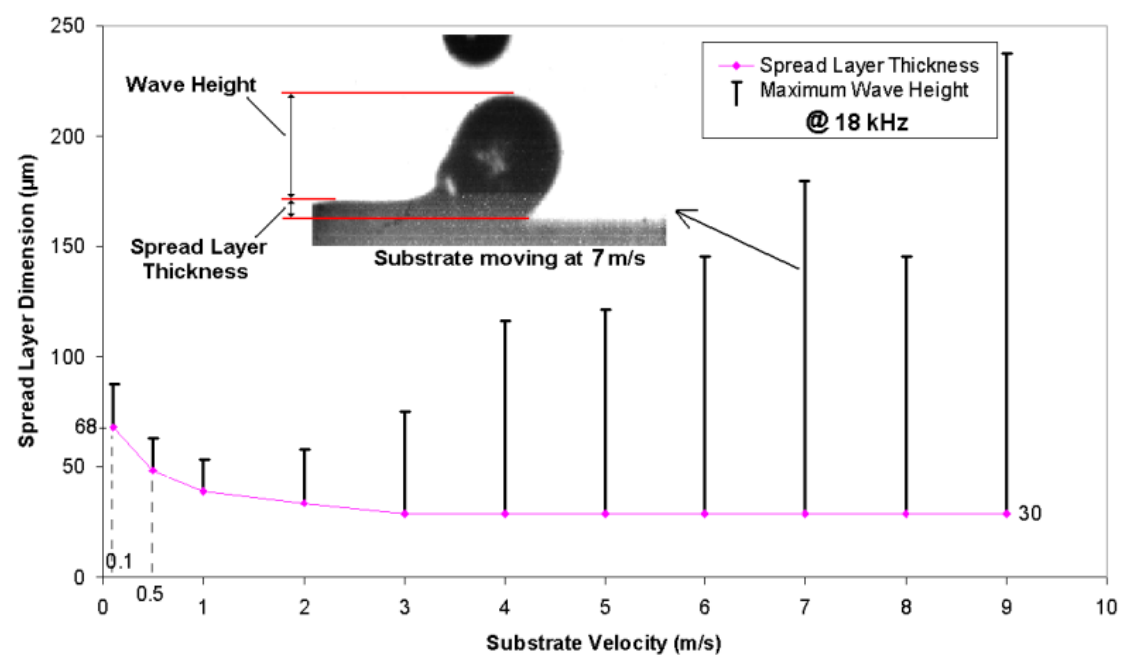

FIGURE 12. Variation of the spread layer dimensions (spread layer thickness and maximum wave height) formed by the droplets jetted at $18 \mathrm{kHz}$ impinging onto the surface with varying velocity

\begin{tabular}{|c|c|c|c|}
\hline Impingement frequency: $18 \mathrm{kHz}$ & \multicolumn{3}{|c|}{ Number of droplets forming the wave } \\
\hline Surface Velocity $\left(\mathrm{m} \mathrm{s}^{-1}\right)$ & $1^{\text {st }}$ wave & $2^{\text {nd }}$ wave & $3^{\text {rd }}$ Wave \\
\hline 4 & 38 & 14 & - \\
\hline 5 & 27 & 22 & - \\
\hline 6 & 30 & 26 & 14 \\
\hline 7 & 25 & 36 & 16 \\
\hline 8 & 30 & 52 & - \\
\hline 9 & 48 & 38 & 35 \\
\hline
\end{tabular}

TABLE 3. The number of droplets contributed in the built-up wave formation at the front of the advancing liquid layer visualised at $18 \mathrm{kHz}$ frequency with different surface velocities

\section{Discussion}

The behaviour of a droplet impinging on a surface originates from an interaction between physical properties of the droplet, its kinetic energy and the physical properties of the surface in the case of a stationary surface as reported by Yarin (2006). In the case of a train of droplets impinging onto a moving surface, although the droplets tend to spread in all directions, the deformation process is biased by the motion of the surface as reported by other researchers in the introduction section. 


\subsection{Spread Layer Thickness}

Droplets impinging onto the moving surface make a liquid layer with a specific thickness. Each train of droplets makes a bead and deposition from several nozzles or several swathes of a single nozzle onto the surface produces a layer. With varying jetting frequency and surface motion, the thickness varied as figures 11 and 12 showed. At a fixed jetting frequency and a lower surface velocity, where the deposition rate is high, the droplets result in material overlap giving a wet surface for droplets to impact. Increasing surface velocity decreased overlap with a decrease in the spread layer thickness. A constant value of the thickness eventually occurred (3 m $\mathrm{s}^{-1}$ and more) as seen in figures 11 and 12 . This is in contrast to the decrease in the linear deposition rate which continues to fall with increasing surface speed. As the droplets impinged onto a dry surface at a low deposition rate (see figure 9), it seems that their spread thickness does not continue to be affected by the surface motion and its induced shear force. At higher surface velocities, the physical characteristics of droplets and the surface (mainly the relative surface tension) were the key players in defining the thickness value at a given jetting frequency.

The spread thickness varied with the droplets kinetics at a surface velocity higher than $3 \mathrm{~m} \mathrm{~s}^{-1}$ for the two jetting frequencies. A thicker layer was achieved at $18 \mathrm{kHz}$. This can be seen in figures 11 and 12, where the values are $25 \mu \mathrm{m}$ and $30 \mu \mathrm{m}$ for 12 $\mathrm{kHz}$ and $18 \mathrm{kHz}$ respectively. The characteristics of the droplet which varied with the jetting frequency are shown in table 2. The impact energy of the droplets and their impact energy rate jetted at $12 \mathrm{kHz}$ were higher than $18 \mathrm{kHz}$ which helped produce a thinner liquid layer. This higher value of impact energy was mostly affected by the higher droplet size generated at the lower frequency (figure 5(a)). 


\subsection{Droplet Train/Moving Surface Interaction Regimes}

The deformation of a droplet onto a moving surface depends on the surface motion and is biased by the surface travel. In the case of a train of droplets impinging onto a moving surface with high impingement frequency however, a continuous bead of material was made with an advancing front in the current study. The droplet train/moving surface interaction regimes were found in the advancing front as classified in figure 13 into three main regimes of 1,2 , and 3.
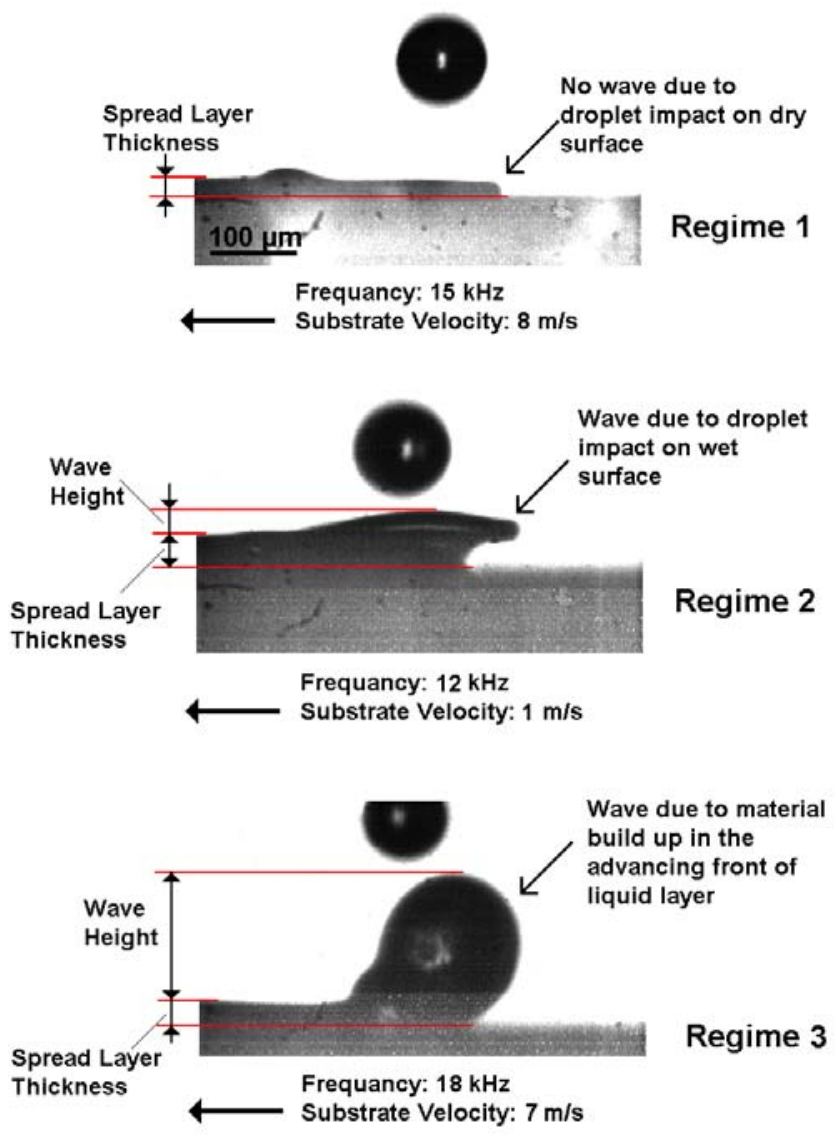

FIGURE 13. Spreading regimes in droplet impact on a moving substrate with different process parameters

\section{Regime 1:}


In Regime 1, no wave is formed at the front of the liquid layer as can be seen in figure 7 and 13. This was generally due to a low linear deposition rate typically lower

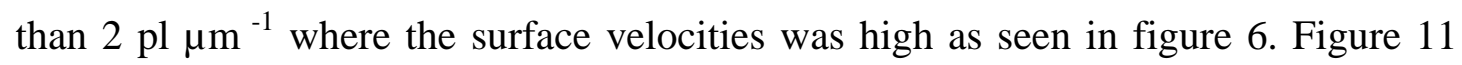
clearly shows the zone of this regime at surface velocities above $5 \mathrm{~m} / \mathrm{s}$.

\section{Regime 2:}

Regime 2, as shown in figure 13, exhibits a crown-like wave at the advancing front of the liquid layer. Generally, this wave was formed with every droplet impingement or every other impingement (figure 8). Therefore, the periodic behaviour of formation and damping of the wave is characteristic of Regime 2. This periodic behaviour could be due to the impingement frequency and the liquid layer thickness. In addition, no secondary droplet(s) from disintegration of the crown like wave was seen in the range of the jetting frequencies and the surface motion. This was predictable from the low Weber and Reynolds numbers extracted from the droplet formation characteristics shown in table 2 .

As shown in figure 8, the source of the crown-like wave was the wet/dry surface boundary. This is due to the difference in surface energy between the spreading solution and the dry (glass) surface where a kinematic discontinuity is made during the deformation process resulting in a crown-like wave. This is similar to the splashing droplets in impingement onto a stationary liquid surface shown by Yarin (2006).

The crown-like wave height was usually smaller than the spread layer thickness. Regime 2 occurred during impingement of the droplets onto the surface at lower velocities where the substrate was made wet by previously spread droplets (substrate velocities less than $3 \mathrm{~m} \mathrm{~s}^{-1}$ in which the deposition rate was lower than $3 \mathrm{pl} \mathrm{\mu m}^{-1}$ ). 
Comparing figures 11 and 12, Regime 2 was seen to have larger waves at lower frequencies which corresponded with higher impact energy of the droplets.

\section{Regime 3:}

Regime 3 was the generation of large waves (as shown in figure 13) at the front of the advancing liquid layer. As the sequence in figure 10 shows, the characteristics of this wave was formation, growth, travel with the advancing liquid layer onto the dry surface and finally separation from the front. The separation was after the wave reached a certain volume when its inertia in association with the surface tension separated it from the advancing front and merged it into the spread liquid layer. This kind of wave had a periodic behaviour with a long time interval from its formation to separation. A number of droplets contributed to the growth of the wave. A typical value was 26 droplets to build-up the wave at $18 \mathrm{kHz}$ frequency with $7 \mathrm{~m} \mathrm{~s}^{-1}$ just before separation from the front.

The height of the wave of Regime 3 was much higher than the wave in Regime 2. In addition, this type of wave build-up was not similar to the crown like wave in Regime 2 in terms of shape. Comparing figures 11 and 12, Regime 3 was seen with higher jetting frequency (18 kHz) and higher surface velocities even though the linear deposition rates of the both jetting frequencies $(12 \mathrm{kHz}$ and $18 \mathrm{kHz})$ were similar (figure 6).

Having the same linear deposition rate and with similar surface velocity, the most interesting question is why no wave was seen at lower jetting frequency categorised as Regime 1 . The main differences between Regime 3 in figure 12 and Regime 1 in figure 11 (surface velocities higher than $3 \mathrm{~m} \mathrm{~s}^{-1}$ ) were the impact energy and the impingement rate of the droplets. In Regime 1 the larger droplets had a higher kinetic 
energy which promoted greater spreading of the material whereas in Regime 3 the droplets were smaller and had lower kinetic energy.

The separation volume inferred from the wave's height increased with surface velocity as shown in figure 12 showing the effect of the surface velocity in Regime 3. Apart from the droplets kinetics differences between the two frequencies of $12 \mathrm{kHz}$ and $18 \mathrm{kHz}$, the time elapsed between two consequent droplets in Regime 3 was much lower than that of Regime 1 (55 $\mu$ s and $83 \mu$ s). In Regime 3, the built-up wave was originated from the excess material which was removed by surface travel from the front before next impinging droplet arrived. Therefore the wave was built-up into a larger wave from the next droplets and the consequent excess material as figure 10 and table 3 revealed. Another contributing factor in the growth process could be the momentum inserting to the left of the wave from the impact of the droplets after the wave was initiated.

It was also of interest to see that the size of the wave increased with surface velocity in Regime 3. This is in despite of the fact that a faster surface should have decreased the excess material left from the last impingement. The important remaining factor could be the surface travel induced shear force. As the applied shear to the wave was in an opposite direction of the surface travel, it assisted the wave build-up process and increased with higher surface velocity as seen in the wave height shown in figure 12. However, to detail the effect of the surface shear force and rate further theoretical investigation is needed.

\section{Conclusions}

The impact phenomena of a train of droplets in different process conditions were discussed in this paper. More specifically, the effect of the droplet formation 
characteristics and the surface motion were correlated with the impact behaviour by considering the linear material deposition rate and droplet impact characteristics. It has been shown that three different regimes occurred during the impact of droplets onto a moving surface. The most dominant factors were the surface velocity and droplet train impingement rate. A higher surface velocity at lower impingement frequency results in a smooth spreading, whereas a high impingement frequency could result in formation of a large built-up wave at the front of the advancing liquid layer.

With deposition of fluid, the formation of the large wave at the front of the spread liquid layer could affect the surface of the deposited layer. After adding several layers, the surface could be bumpy affecting the fabricated part's quality and deteriorating the outer geometry of the part. Therefore, the current study suggests a use of lower jetting frequencies and higher surface velocities for a better surface finish and improved geometrical and dimensional accuracy in a jetting-based additive manufacturing process.

\section{References}

Calvert, P., 2001. Inkjet printing for materials and devices. Chem. Mater. 2001, 13, 3299-3305.

Chen, R.H., Wang, H.W., 2005. Effects of tangential speed on low-normal-speed liquid drop impact on a non-wettable solid surface. Exp. Fluid. 39, 754-760.

Courbin, L., Bird, J.C., Stone, H.A., 2006. Splash and anti-splash: Observation and design. CHAOS. 16, 041102.

Frohn, A., Roth, N., 2000. Dynamics of Droplets, Springer, Berlin, Germany, pp. 151177. 
Gritts, A., Varero, R., Jones, C.L., Dolinsek, S., 2006. A Concept of manufacturing system enabling the creation of custom-fit products. Proceedings of $10^{\text {th }}$ International Research/Expert Conference on Trends in the Development of Machinery and Associated Technology. Barcelona, Spain.

Hopkinson, N., Hague, R.J.M. and Dickens, P.M., 2006. Rapid Manufacturing: An Industrial Revolution for the Digital Age, John Wiley and Sons, West Sussex, UK, pp. 58-63.

Le, H.P., 1998. Progress and trends in ink-jet printing technology. J. Imag. Sci. Tech. 42, 42-69.

Leneweit, G., Koehler, R., Roesner, K.G., Schaefer, G., 2005. Regimes of drop morphology in oblique impact on deep fluids. J. Fluid Mech. 543, 303-331.

Mundo, C., Sommerfeld, M., Tropea, C., 1995. Droplet-wall collisions: experimental studies of the deformation and breakup process. Int. J. Multiphas. Flow. 21, 151173.

Okawa, T., Shiraishi, T., Mori, T., 2008. Effect of impingement angle on the outcome of single water drop impact onto a plane water surface. Exp. Fluid. 44, 331-339.

Rayleigh, J.W.S., 1878. On the instability of jets. Proc. London Math. Soc. 10, 4-13.

Rein, M., 2002. Drop-Surface Interactions, Springer-Wien, New York, pp. 25-39.

Rioboo, R., Bauthier, C., Conti, J., Voue, M., De Coninck, J., 2003. Experimental investigation of splash and crown formation during single drop impact on wetted surfaces. Exp. Fluid. 35, 648-652.

Yarin, A.L., 2006. Drop impact dynamics: splashing, spreading, receding, bouncing. Annu. Rev. Fluid Mech. 38, 159-192. 
Yarin, A.L., Weiss, D.A., 1995. Impact of drops on solid surfaces: self-similar capillary waves, and splashing as a new type of kinematic discontinuity. J. Fluid Mech. 283, 141-173. 